\title{
Validación de dos activadores prebióticos en el desarrollo de terneras medias Ayrshire sobre los 3,000 m.s.n.m. en Sangolquí, Pichincha
}

\section{Validation of two prebiotic activators in the development of crossbred ayrshire heifers, 3,000 meters above the sea level in Sangolquí, Pichincha}

\author{
Luis Miguel Medina ${ }^{1}$, Galo Jacho ${ }^{1}$, Lenin Ron ${ }^{1}$, Jorge Caicedo ${ }^{1}$ \\ I Universidad Central del Ecuador. Facultad de Ciencias Agrícolas. Carrera de Ingeniería Agronómica. Jerónimo Leiton y Av. La Gasca s/n. \\ Ciudadela Universitaria. 170521 Quito, Ecuador
}

\begin{abstract}
Resumen
En la provincia de Pichincha, cantón Rumiñahui, parroquia Rumipamba, sobre los 3,000 m.s.n.m., se llevó a cabo la validación de dos activadores prebióticos en terneras medias Ayrshire. Para la fase de campo se emplearon terneras puras con una edad de entre 4-6 meses de edad. Las variables dependientes fueron: el incremento de peso, altura, diámetro de cinchera y capacidad corporal; los datos se registraron cada 15 días. Se empleó un diseño completamente al azar (DCA) con covariables, el mismo que estuvo conformado por tres tratamientos y cinco observaciones para cada tratamiento, cada ternera fue una unidad experimental. De acuerdo al análisis de varianza realizado para cada variable evaluada se observa que no existen diferencias significativas entre los tratamientos; pero para el análisis de la covariable el ANOVA detectó diferencias significativas para la interacción de cada variable.
\end{abstract}

Palabras clave: terneras, covariable, ayrshire, crianza a gran altura.

\begin{abstract}
The validation of two prebiotic activators in crossbred Ayrshire heifers was conducted in the province of Pichincha, Rumiñahui County, Rumipamba Parish, at 3,000 meters a.s.1. For the field phase, purebred Ayrshire heifers with an average age of 4 to 6 months old were studied and data on their weight, height and waist diameter increase, as well as their body capacity were measured every two weeks. An experimental design (DCA) with covariables, the age being the covariable, consisted of three treatments and five observations for each treatment. The experimental unit was the heifer. According to the analysis of variance conducted for each tested variable, there are no significant differences between treatments; however, for the analysis of the covariable (ANOVA), significant differences were detected for the interaction of each variable.
\end{abstract}

Keywords: heifers, covariable, ayrshire, high altitude breeding. 


\section{Introducción}

La producción de leche es un rubro importante de la canasta agrícola delEcuador. De acuerdo al MAGAP (2014) este segmento movilizó alrededor de 700 millones de dólares en el 2014. Factores como el crecimiento demográfico y los acelerados procesos de urbanización han desplazado a la ganadería de leche a zonas por encima de los 3,000 m.s.n.m., lo que implica nuevos retos para la producción de leche en la región andina. En este contexto, las explotaciones ganaderas buscan nuevas formas de manejo de sus hatos en unas condiciones que no son las ideales para la crianza de ganado lechero.

Por estas razones el ganadero se ha visto en la necesidad de buscar nuevos productos que promuevan el desarrollo óptimo de los animales. Una de estas alternativas es el uso de prebióticos que son compuestos que el animal no digiere, pero que tienen un efecto fisiológico en el intestino al estimular, de manera selectiva el incremento de la actividad de las bacterias beneficiosas en el intestino del animal (Zbiden, 2008).

Las dosis de prebióticos en animales muy jóvenes o muy viejos deben permanecer en niveles bajos para no cambiar drásticamente la digestibilidad, es por esto que los animales jóvenes, en este caso las terneras, requieren dietas altamente digestibles para mantener las tasas de crecimiento aceptables. Pese a esto, son escasas las investigaciones que validen el uso de estos compuestos a la realidad local.

Con estos antecedentes, esta investigación se centra en el uso de prebióticos en la alimentación de terneras medias Ayrshire, validando la respuesta de los prebióticos en su desarrollo y demostrar si existen nuevas alternativas nutricionales que mejoren considerablemente el desarrollo de las terneras sobre los 3,000 m.s.n.m.

\section{Materiales y métodos}

\subsection{Ubicación}

La presente investigación se realizó en la hacienda "Wagra Cacharina" (El Páramo), localizada en la parroquia Rumipamba, cantón Rumiñahui Pichincha a una altura promedio de 3,200 m.s.n.m. con una temperatura media anual de $9{ }^{\circ} \mathrm{C}$ y una pre- cipitación media anual de $1,496.8 \mathrm{~mm}$. al año. Se inició con el ensayo el 26 de enero del 2015.

\subsection{Variables}

Las variables en estudio fueron: el incremento de peso corporal, la altura a la cruz, el incremento de cinchera y la condición corporal. El incremento de peso corporal se midió con una cinta bovino métrica a la altura de la cinchera. La altura a la cruz se evaluó tomando la altura en centímetros con una regla graduada. El incremento de cinchera se evaluó tomando el diámetro de cinchera con una cinta bovino métrica. En tanto que la condición corporal fue evaluada a partir del inicio de la investigación con base en una escala que va de uno a cinco. La recolección de datos tuvo lugar cada 15 días a partir de que los prebióticos fueron administrados.

\subsection{Manejo del experimento}

El experimento estuvo conformado por tres tratamientos los cuales se especifican en la Tabla 1. Para identificar a las terneras de cada tratamiento se utilizaron collares de color verde para el testigo, verde, rojo para el tratamiento 1 (prebiótico 1) y azul para el tratamiento 2 (prebiótico 2. La alimentación y la recolección de datos se realizaron en un corral pequeño con una manga, lo que facilitó el manejo de las terneras durante la investigación. Para el análisis estadístico se utilizó un diseño completamente al azar con covariable, siendo la covariable la edad de las terneras.

\section{Resultados y discusión}

\subsection{Incremento de Peso Corporal}

El análisis de varianza (Tabla 2) realizado para el estudio del incremento de peso, muestra que no existen diferencias significativas entre los tratamientos. Una posible explicación para este resultado es que la alimentación administrada a cada tratamiento (balanceado, prebióticos, sales minerales y pasto a voluntad) no influyó mayoritariamente en la variable incremento de peso. De acuerdo a Wattiaux (1998) al presentar una mayor exigencia energética en la altura, los animales tienden a metabolizar rápidamente el alimento administrado.

En esta investigación se observó que sobre los 3,000 m.s.n.m. los animales presentaron un mayor 
requerimiento energético, lo que puede ser apreciable al observar que el incremento de peso diario fue menor a 800 - $900 \mathrm{~g}$ /día lo que hizo que no presenten diferencias significativas en la ganancia de peso entre tratamientos (Lanuza, 2008). Por su parte, Barra (2005) menciona que dado los altos requerimientos energéticos de los animales destinados a la producción intensiva de leche, el principal componente de la dieta lo constituye el concentrado (energía más proteína más sales minerales).

Tabla 1. Tratamientos aplicados.

\begin{tabular}{|c|c|}
\hline Tratamientos & Descripción \\
\hline Tratamiento 1 & $1 \mathrm{~kg}$ de balanceado $+10 \mathrm{gr}$ de sales minerales + pasto a voluntad + agua a voluntad. \\
\hline Tratamiento 2 & $\begin{array}{l}1 \mathrm{~kg} \text { de balanceado } 1+7 \mathrm{gr} \text { del prebiótico } 1+10 \mathrm{gr} \text { de sales minerales }+ \text { pasto a voluntad }+ \\
\text { agua a voluntad. }\end{array}$ \\
\hline Tratamiento 3 & $\begin{array}{l}1 \mathrm{~kg} \text { de balanceado } 1+25 \text { gr del prebiótico } 2+10 \text { gr de sales minerales }+ \text { pasto a voluntad }+ \\
\text { agua a voluntad. }\end{array}$ \\
\hline
\end{tabular}

En el análisis de la covariable (peso inicial), el ANOVA detectó diferencias significativas para la interacción entre el peso inicial sobre el peso final, indicando que el primero influye significativamente sobre el peso final de los animales. Los leves incrementos observados en los animales se debieron al peso inicial de los mismos y no al efecto de los tratamientos.

La respuesta visible que presenta el testigo se debe a que el balanceado empleado posee también suplementos de vitaminas $\mathrm{AD} 3 \mathrm{E}$, lo que hace que el balanceado esté diseñado como un promotor del desarrollo de las terneras que permite mejorar la ganancia de peso durante la crianza y alcanzar tempranamente el peso óptimo para el primer servicio (Lanuza, 2008).

De acuerdo a Lanuza (2008), mientras más avanzada es la edad, el aumento de peso en el animal adulto estará representado por una mayor proporción de grasa en la composición química de la ganancia de peso. Por el contrario, en el animal joven, esto estará dado por una mayor proporción de proteína (músculo) en desmedro de la grasa.

Tabla 2. Análisis de varianza para el incremento de peso $(\mathrm{kg})$, para la validación de dos activadores prebióticos en terneras medias Ayrshire sobre los 3,000 m.s.n.m.

\begin{tabular}{lcccrr}
\hline \multicolumn{1}{c}{ Fuente de variación } & Suma de cuadrados & gl & $\begin{array}{c}\text { Cuadrado } \\
\text { medio }\end{array}$ & F & p-value \\
\hline Modelo & $22,611.46$ & 3 & $7,537.15$ & & 0.000 \\
Tratamiento & 25.97 & 2 & 12.98 & 151.22 & 0.775 \\
Peso inicial & $22,076.93$ & 1 & 22.076 & 0.26 & 0.000 \\
Error & 548.27 & 11 & 49.48 & & \\
Total & $23,159.73$ & 14 & & & \\
\hline $\mathrm{R}^{2}$ & $\mathrm{R}^{2}$ Ajustada & $\mathrm{CV}$ & & \\
0.98 & 0.97 & $4.42 \%$ & & & \\
\hline
\end{tabular}

\subsection{Incremento Altura a la Cruz}

La Tabla 3 muestra que no existen diferencias significativas entre tratamientos para la variable altura a la cruz. De acuerdo a Gallardo (2000) los aditivos (prebióticos) para mejorar la eficiencia de la conversión de los alimentos se debería considerar que al reemplazar un insumo por otros ingredientes de menor desempeño nutricional o cuando se desea hacer un uso intensivo y eficiente de forrajes en las dietas, estos generaran una respuesta del animal a incrementar su potencial fisiológico en este caso la altura a la cruz.

En el análisis de la covariable (altura inicial), el ANOVA detectó diferencias significativas para la interacción entre la altura inicial sobre la altura final, 
indicando que el primero influye significativamente sobre la altura final de los animales. De la misma manera, como en el caso del peso final, los leves incre- mentos observados en la altura final se debieron al uso de la altura inicial como covariable y no a la aplicación de los tratamientos.

Tabla 3. Análisis de varianza para el incremento de Altura a la cruz, para la validación de dos activadores prebióticos en terneras medias Ayrshire sobre los 3,000 m.s.n.m. en Sangolquí.

\begin{tabular}{lccrrl}
\hline \multicolumn{1}{c}{ Fuente de variación } & Suma de cuadrados & gl & $\begin{array}{c}\text { Cuadrado } \\
\text { medio }\end{array}$ & F & p-value \\
\hline Modelo & $1,055.46$ & 3 & 351.82 & & \\
Tratamiento & 0.99 & 2 & 0.49 & 0.11 & 0.90 \\
Peso inicial & $1,051.93$ & 1 & $1,051.75$ & 224.85 & 0.000 \\
Error & 51.45 & 11 & 4.68 & & \\
Total & $1,106.90$ & 14 & & & \\
\hline $\mathrm{R}^{2}$ & $\mathrm{R}^{2}$ Ajustada & $\mathrm{CV}$ & & \\
0.98 & 0.97 & $2.27 \%$ & & & \\
\hline
\end{tabular}

\subsection{Incremento de Cinchera}

El análisis de varianza para esta variable (Tabla 4) muestra que, al igual que en los casos anteriores, no existen diferencias entre tratamientos. En el análisis de la covariable (incremento de cinchera inicial), el ANOVA detectó diferencias significativas para la interacción entre el incremento de cinchera inicial sobre el incremento de cinchera final, indicando que el primero influye significativamente sobre el incremento de la cinchera final de los animales. Es importante mencionar, que el diámetro de la cinchera está estrechamente correlacionado con el peso de los animales. Es por esta razón que los leves incrementos observados en la cinchera final se debieron al uso de la cinchera inicial como covariable y no a la aplicación de los tratamientos.

Para Bath (1978) los nutrientes que necesita la ternera para incrementar el diámetro de la caja torácica son energía metabolizable, proteína, minerales, vitaminas y agua; de estos el primero y el último contribuyen mayormente sobre el incremento del diámetro de la cinchera. Por ello, el testigo, sin necesidad de ningún aditivo, tuvo una mejor respuesta ante el desarrollo fisiológico de la ternera, debido a que las terneras fueron alimentadas con el balanceado que poseía un $16 \%$ de proteína cruda.

\subsection{Capacidad Corporal}

Dado que esta variable es de naturaleza cualitativa ordinal y, por tanto, no presenta una distribución normal, se aplicó una prueba de Kruskal Wallis, la cual es útil en casos en que los preceptos de normalidad no se cumplen.

Al aplicar la prueba de Kruskal Wallis se observa que el valor $\mathrm{p}$ es mayor a 0.05 , por lo que no es posible rechazar la hipótesis nula de que los tratamientos tuvieron una respuesta similar. Es decir, al igual que en las variables cuantitativas analizadas anteriormente, la adición de aditivos prebióticos no implica una respuesta mejor que la obtenida a partir del tratamiento testigo.

Tabla 4. Análisis de varianza para el incremento de Cinchera, para la validación de dos activadores prebióticos en terneras medias Ayrshire sobre los 3,000 m.s.n.m. en Sangolquí.

\begin{tabular}{lccccc}
\hline \multicolumn{1}{c}{ Fuente de variación } & Suma de cuadrados & gl & $\begin{array}{c}\text { Cuadrado } \\
\text { medio }\end{array}$ & F & p-value \\
\hline Modelo & $1,743.55$ & 3 & 581.18 & 140.87 & 0.000 \\
Tratamiento & 2.39 & 2 & 1.20 & 0.29 & 0.753 \\
Peso inicial & 1706.62 & 1 & $1,706.62$ & 413.6 & \\
Error & 45.38 & 11 & 4.13 & & \\
Total & $1,788.93$ & 14 & & \\
\hline $\mathrm{R}^{2}$ & $\mathrm{R}^{2}$ Ajustada & $\mathrm{CV}$ & & \\
0.97 & 0.97 & $1.67 \%$ & & \\
\hline
\end{tabular}


Tabla 5. Prueba de Kruskal Wallis para Capacidad Corporal, Para la validación de dos activadores prebióticos en terneras medias Ayrshire sobre los 3,000 m.s.n.m. en Sangolquí.

\begin{tabular}{|c|c|c|c|c|c|c|c|c|c|}
\hline Variable & Tratamiento & $\mathbf{N}$ & Medias & D.E. & Promedio & gl & $\mathbf{C}$ & $\mathbf{H}$ & p-value \\
\hline Capacidad Corporal Final & Prebiótico 1 & 5 & 3.40 & 0.22 & 6.80 & 2 & 0.77 & 0.72 & 0.6271 \\
\hline Capacidad Corporal Final & Prebiótico 2 & 5 & 3.50 & 0.35 & 8.00 & & & & \\
\hline Capacidad Corporal Final & Testigo & 5 & 3.60 & 0.42 & 9.20 & & & & \\
\hline
\end{tabular}

\section{Conclusiones}

Este estudio analizó el efecto de los prebióticos sobre las variables: incremento de peso, altura a la cruz, diámetro de cinchera y capacidad corporal. Los resultados no reflejaron diferencias significativas, demostrando que el uso de prebióticos en la suplementación de terneras sobre los 3,000 m.s.n.m. no dio los resultados esperados. Debido a la diferencia de edades entre las terneras (4-6 meses) al inicio de la investigación se empleó una variable correctora, en este caso la covariable (edad), lo que controló los efectos de la diferencia de edad en las variables bajo análisis.

Más allá de la lectura e interpretación de estos resultados, el aporte más importante de esta inves-

\section{Referencias}

Almeyda, J. (2013). Recría de animales de reemplazo en sistemas intensivos. Lima, Perú: Universidad Nacional La Molina

Ayón M., Cueva. (1998) Adaptación del Ganado Bovino a la altura. Lima, Perú: Facultad de Medicina Veterinaria de la UNMSM. Pub. Tec. $N^{\circ} 38$

Barra, F. (2005). Manejo de la Alimentación de Animales a Corral, Argentina: disponible en URL: www.produccion-animal.com.ar [Consultado 25 de Abril 2015]

Bargo, F. (2005). Evaluación del Estado Corporal en vacas lecheras. Informe Técnico Elanco Animal Health. Argentina: S.n., disponible en URL: www.produccion-animal.com.ar [Consultado 3 de Marzo 2015]

Gallardo, M. (2000). Concentrados y Subproductos para la Alimentación de Rumiantes. Argentina: INTA s.n. Boletín $\mathrm{N}^{\circ} 43$.

Gibson, G., y Roberfroid, M. (2008). Handbook of prebiotic. Minesota, U.S.A.: Editorial Gibson, G. y Roberfroid”. tigación es la conclusión de que el uso de prebióticos a alturas mayores de 3,000 m.s.n.m. no ofrece los resultados esperados, por tanto, en estas condiciones, la inversión en este tipo de aditivos no se justifica. Sin embargo, es preciso resaltar que se requiere un mayor número de estudios en diferentes ambientes para probar esta hipótesis de manera más concluyente.

Por otro lado, todos los análisis de varianza reportaron un efecto significativo para la variable correctora que controlaba la edad de las terneras, por tanto, el uso de variables de control en este tipo de estudio resulta esencial a fin de obtener mayor robustez en los resultados.

Hazard, S., (1996). Alimentación de terneros y vaquillas de lechería. Colombia: INIA.

Lanuza, F. (2008). Crianza de Terneros y Reemplazos de lechería. Argentina: Instituto de Investigaciones Agropecuarias. Boletín INIA N 148 .

Ministerio de Agricultura, Acuicultura y Pesca. (2014). Desarrollo del sector ganadero en el último año. Quito, Ecuador: MAGAP.

Wattiaux, M. (2000). Crianza de terneras desde el nacimiento hasta el destete. Madison, U.S.A.: Instituto Babcock para la Investigación y Desarrollo Internacional de la Industria Lechera". Universidad de Winsconsin Madison.

Zbiden, E. (2008). Uso de prebióticos en la alimentación. Chile: "Revista tecno vet" Facultad de Ciencias Veterinarias y Pecuarias Universidad de Chile. Santiago de Chile, Chile. 